\title{
Is there any feedback between AGN and star-forming activity in radio-selected AGN?
}

\author{
Manuela Magliocchetti* \\ IAPS-INAF, Via Fosso del Cavaliere 100, Rome, Italy \\ E-mail: manuela@iaps.inaf.it
}

In order to investigate the FIR properties of radio-active AGN, we have considered four different fields where both radio and FIR observations are the deepest to-date: COSMOS, GOODS-South, GOODS-North and the Lockman Hole. Out of a total of 574 radio-selected AGN, $40 \%$ are found to have a counterpart in Herschel maps. The percentage is maximum in the GOODS-North (72\%) and minimum (36\%) in COSMOS, where FIR observations are shallower. Our study shows that in all cases FIR emission is associated to star-forming activity within the host galaxy. Such an activity can even be extremely intense, with star-forming rates as high as $\sim 10^{3}-10^{4} \mathrm{M}_{\odot} \mathrm{yr}^{-1}$. AGN activity does not inhibit star formation in the host galaxy, just as on-site star-formation does not seem to affect AGN properties, at least those detected at radio wavelengths and for $z \gtrsim 1$. Given the very high rate of FIR detections, we stress that this refers to the majority of the sample: most radio-active AGN are associated with intense episodes of star-formation. However, the two processes proceed independently within the same galaxy, at all redshifts but in the local universe, where powerful enough radio activity reaches the necessary strength to switch off the on-site star formation. Our data also show that for $\mathrm{z} \gtrsim 1$ the hosts of radio-selected star-forming galaxies and AGN are indistinguishable from each other both in terms of mass and IR luminosity distributions. The two populations only differentiate in the very local universe, whereby the few AGN which are still FIR-active are found in galaxies with much higher masses and luminosities.

EXTRA-RADSUR2015 (*)

20-23 October 2015

Bologna, Italy

(*) This conference has been organized with the support of the Ministry of Foreign Affairs and International Cooperation, Directorate General for the Country Promotion (Bilateral Grant Agreement ZA14GR02 - Mapping the Universe on the Pathway to SKA)

\footnotetext{
* Speaker.
} 


\section{The Fields}

The VLA-COSMOS Large Project observed the $2 \mathrm{deg}^{2}$ of the COSMOS field at $1.4 \mathrm{GHz}$. The catalogue adopted here is that presented in [1], which comprises 2382 sources selected above an integrated flux of $60 \mu \mathrm{Jy}$. Redshifts for 1537 of these sources (corresponding to $\sim 65 \%$ of the parent population), come from the [2] catalogue.

Radio sources in the Lockman Hole field come from the 1.4 GHz observations of [3]. The sensitivity of these observations was $\sigma \sim 6 \mu \mathrm{Jy} \mathrm{beam}^{-1}$. This leads to a catalogue of 1303 unique sources, selected to have a peak-to-local noise greater than 5 . Redshifts for 723 of these objects (corresponding to $55.7 \%$ of the original radio sample on the overlapping area between radio and optical/near infrared observations) have been obtained from the [4] catalogue.

Deep, 1.4 GHz, radio observations of the GOODS-North field (hereafter GOODS-N) are presented in [5]. These authors provide a catalogue of 1230 discrete radio sources above a $5 \sigma$ detection threshold of $\sim 20 \mu \mathrm{Jy}$ at the field centre. We then cross-correlated the radio catalogue with that of optically-selected sources used in [6]. The number of radio objects which fall within the area observed at the other wavebands is 401 . Out of these, 267 , corresponding to $\sim 67 \%$ of the parent radio catalogue, are endowed with a redshift determination.

Deep, $1.4 \mathrm{GHz}$, radio observations of the whole Extended Chandra Deep Field South (ECDFS) are presented in [7]. A smaller area, corresponding to what is called the GOODS Southern Field (hereafter GOODS-S), is provided with high quality photometric and spectroscopic observations [8]. By cross-correlating these two catalogues, we obtain that out of the 142 radio sources which fall within the GOODS-S area, 111 (corresponding to $\sim 78 \%$ of the parent catalogue) possess a redshift determination.

The COSMOS, Lockman Hole, GOODS-N and GOODS-S fields have all been observed by the PACS instrument onboard of the Herschel satellite at both $100 \mu \mathrm{m}$ and $160 \mu \mathrm{m}$. The PEP survey [9] provides catalogues for all sources in these fields at different Far Infrared flux limits, COSMOS being the shallowest and the two GOODS fields being the deepest ones.

\section{AGN selection via radio luminosity}

The method adopted here was introduced in [10] and [11] and is based on the results of [12] who provide radio luminosity functions for the two classes of radio-selected AGN and star-forming galaxies up to redshifts $\sim 2.5$. Investigations of their results show that the radio luminosity $\mathrm{P}_{\text {cross }}$ beyond which AGN-powered galaxies become the dominant radio population scales with redshift roughly as

$$
\log _{10} P_{\text {cross }}(z)=\log _{10} P_{0, \text { cross }}+z
$$

at least up to $z \sim 1.8 . P_{0, \text { cross }}=10^{21.7}\left[\mathrm{~W} \mathrm{~Hz}^{-1} \mathrm{sr}^{-1}\right]$ is the value which is found in the local universe and which roughly coincides with the break in the radio luminosity function of star-forming galaxies (cfr [13]). Beyond this value, their luminosity function steeply declines, and the contribution of star-forming galaxies to the total radio population is drastically reduced to a negligible percentage. The same trend is true at higher redshifts, and since the radio luminosity function of star-forming galaxies drops off in a much steeper way than that of AGN at all $z$, we expect the 
chances of contamination in the two populations to be quite low. We then distinguished between AGN-powered galaxies and star-forming galaxies by means of equation (2.1) for $z \leq 1.8$ and by fixing $\log _{10} \mathrm{P}_{\text {cross }}(\mathrm{z})=23.5\left[\mathrm{~W} \mathrm{~Hz}^{-1} \mathrm{sr}^{-1}\right.$ ] at higher redshifts. This procedure identifies $482 \mathrm{AGN}$, corresponding to $\sim 33 \%$ of the parent sample, and 1026 star-forming galaxies in the COSMOS field, 357 star-forming galaxies and 45 AGN (corresponding to $13 \%$ of the total radio population) in the Lockman Hole, 89 star-forming galaxies and 15 AGN (corresponding to $13 \%$ of the total radio population) in the GOODS-S, $235 \mathrm{SF}$ and 32 AGN (corresponding to $12 \%$ of the total radio population) in the GOODS North. We note that the different percentages are due to the different flux limits of the radio surveys: brighter surveys such as the COSMOS-VLA probe a higher percentage of AGN. We also note that, due to the adopted selection criteria and thanks to the great depths of the considered surveys, all the AGN samples (except for COSMOS which is complete up to $z \sim 3.5$ ) are complete with respect to radio selection at all probed redshifts, i.e. the considered samples include all radio-emitting AGN endowed with a redshift determination.
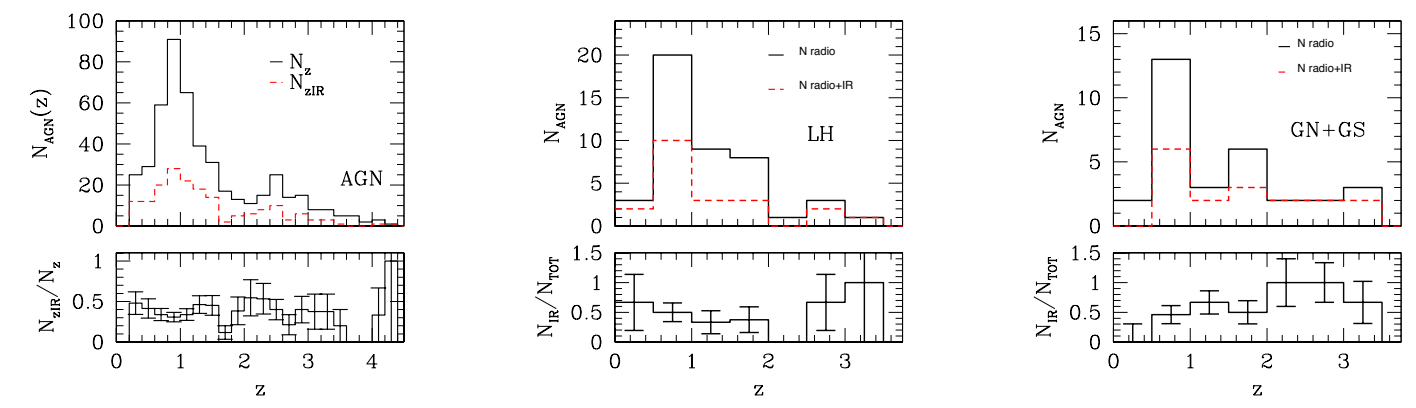

Figure 1: Top panels: distribution of radio-emitting AGN as a function of redshift. From left to right, the panels show sources in the COSMOS field, Lockman Hole (LH) and in the combined GOODS-N and GOODS-S fields (GN+GS). The solid lines indicate the whole population of radio-emitting AGN selected on the basis of their radio luminosity, while red dashed lines show the subsets which also have counterparts at FIR wavelengths. Bottom panels: fraction of radio emitting AGN with a FIR counterpart as a function of redshift.

We then looked for counterparts for these sources in the Herschel maps. It turns out that 175 AGN from the COSMOS-VLA Survey emit at either $100 \mu \mathrm{m}$ or $160 \mu \mathrm{m}$. The same is true for 21 AGN in the Lockman Hole, 23 in the GOODS-N and 8 in the GOODS-S. A point which is worth stressing is the strong dependence of the fraction of radio-emitting AGN which are found to possess a counterpart in the Herschel maps as a function of limiting infrared flux. In fact, one goes from the $\sim 40 \%$ of the COSMOS field to the $\sim 50 \%$ of the Lockman Hole and GOODS-S, to the $72 \%$ of the GOODS-N. These figures only depend on the different depths of Far Infrared (hereafter FIR) observations on the different fields. Allowing for the relatively large uncertainties due to the small areas covered, these results imply that the overwhelming majority $(66 \pm 12 \%$, percentage which, as already seen, rises to $\sim 72 \%$ in the GOODS-N) of radio-emitting AGN in the GOODS-S and GOODS-N are imaged at FIR wavelengths down to fluxes $0.6 \mathrm{mJy}$ at $100 \mu \mathrm{m}$ and $1.3 \mathrm{mJy}$ at 160 $\mu \mathrm{m}$. This is a rather surprising result, since - at least locally - AGN selected at radio wavelengths have always being associated with passive galaxies, with little or no ongoing stellar activity. 
Another remarkable point that emerges from our analysis is that deeper FIR observations do not pick up any new population nor they do single out any different property of the considered population. In fact, the only effect of increased FIR depth is that of evenly increasing the number of sources which are also FIR emitters, independent of look-back time (cfr Figure 1), radio flux and (cfr [10]; [11]) other physical parameters which characterize the sources under examination.

\section{Results: 1) Properties of the inner engine.}
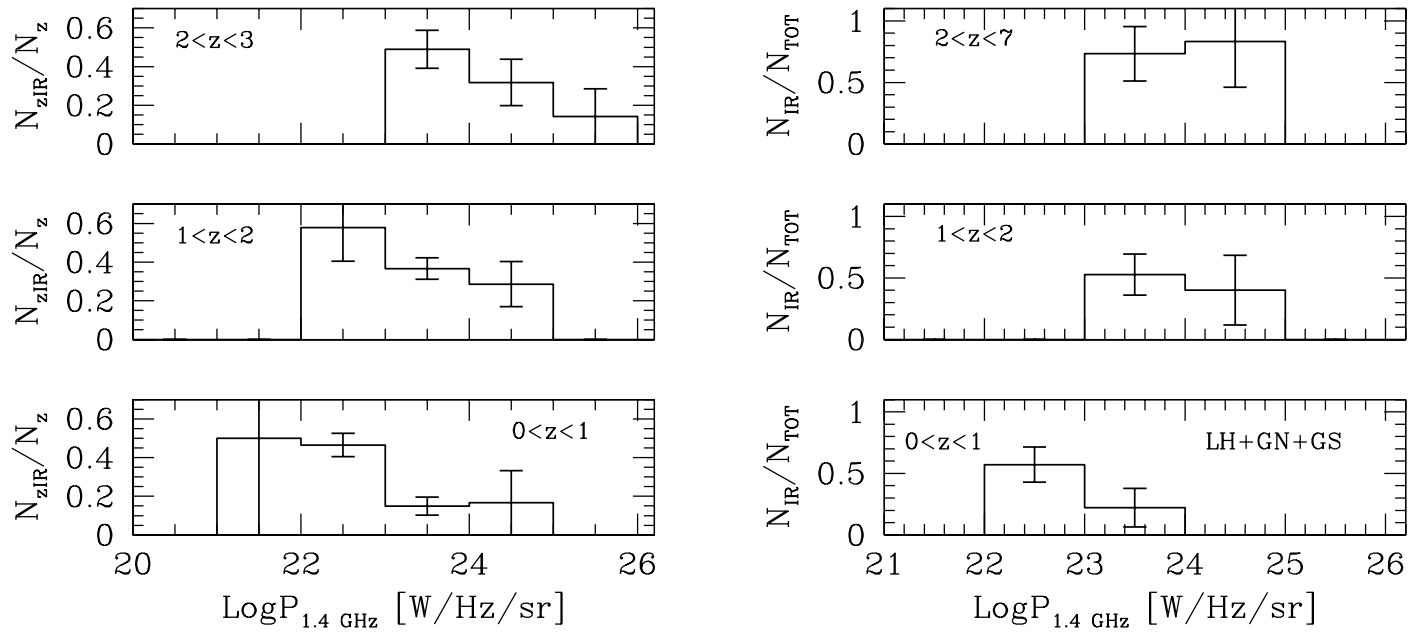

Figure 2: Fraction of radio-selected AGN with FIR emission as a function of radio power in three different redshift bins. The left-hand panel corresponds to the COSMOS field, while the right-hand one to the combined Lockman Hole + GOODS-N + GOODS-S.

Important information on the inner engine of radio-emitting AGN and assessment on whether there are any differences in the presence of FIR emission can be gathered by investigation of the distributions of their radio luminosities both in the presence and in absence of a FIR signature. Figure 2 shows the fraction of FIR-emitting AGN as a function of radio luminosity in three different redshift bins. The left-hand panel refers to COSMOS while, in order to increase the statistical significance of the results, in the right-hand panel we have plotted the same quantity for all the three Lockman Hole, GOODS-N and GOODS-S fields combined together. Two main features can be appreciated from the plots. The first one is that, irrespective of the depth of FIR observations, in the local universe FIR emission is only suppressed in very powerful, $\mathrm{P}_{1.4 \mathrm{GHz}} \gtrsim 10^{23}\left[\mathrm{~W} \mathrm{~Hz}^{-1}\right.$ $\mathrm{sr}^{-1}$ ], AGN. In fact, about $50 \%$ of less luminous AGN are observed to emit at FIR wavelengths. At higher redshifts instead, one has that even powerful radio AGN have augmented chances for being FIR emitters. Indeed, at high enough redshifts and for deep enough FIR observations (cfr right-hand plot of Figure 2) we find that the overwhelming majority of radio-selected AGN of all radio luminosities emits at FIR wavelengths. What one can then conclude from this analysis is that chances for an AGN which is active at radio wavelengths to also emit at FIR wavelengths are maximum at high $(z \gtrsim 1)$ redshifts. For deep enough FIR observations such as those performed on the 

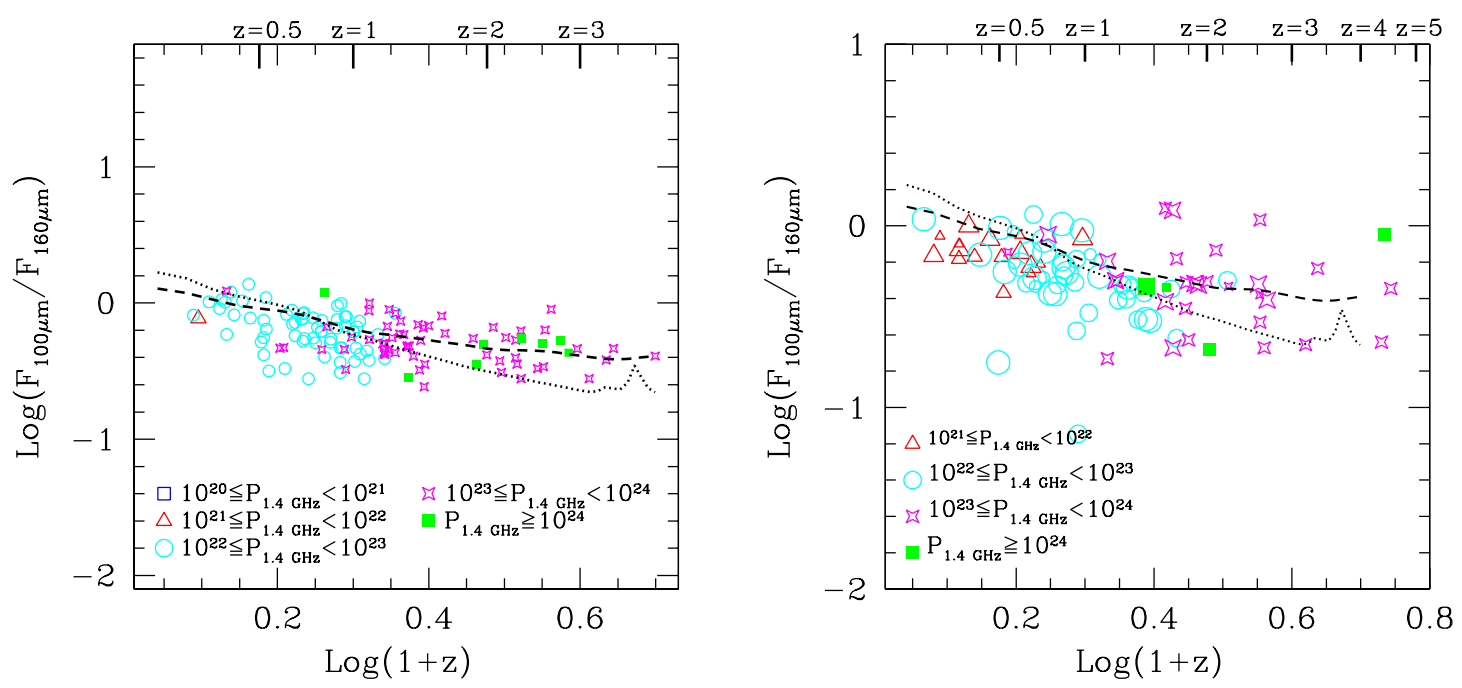

Figure 3: Ratio between $\mathrm{F}_{100 \mu \mathrm{m}}$ and $\mathrm{F}_{160 \mu \mathrm{m}}$ fluxes as a function of redshift for radio-selected sources classified as AGN and with a FIR counterpart in the PEP survey. Different symbols correspond to different intervals in radio luminosity (measured in $\left[\mathrm{W} \mathrm{Hz} \mathrm{sr}^{-1}\right]$ ). The dashed lines represent the trend obtained for the SED of M82, while the dotted ones correspond to Arp220. Data in the the left-hand panel refer to the COSMOS field, while those in the right-hand panel to the Lockman Hole (large symbols), GOODS-N (intermediate-size symbols) and GOODS-S (small symbols) combined together.

GOODS fields, this probability reaches the value of $\sim 100 \%$, independent of radio luminosity. This implies that almost all radio-selected AGN at $z \gtrsim 1$ are also the sites of FIR emission. The situation changes in the more local, $z \lesssim 1$, universe, as in this latter case one finds that the relative fraction of FIR-active AGN is lower and, most importantly, decreases as a function of radio luminosity. In other words, in the local universe mostly low-luminosity AGN are found to be associated with FIR emission. Only very few higher-power, $\mathrm{P}_{1.4 \mathrm{GHz}} \gtrsim 10^{23} \mathrm{~W} \mathrm{~Hz}^{-1} \mathrm{sr}^{-1}$, sources instead appear on FIR maps. We note that our results agree with the local findings which place radio-emitting AGN inside early-type galaxies, with little or no on-site ongoing star-forming activity. Our data however indicate that this is only true in the local universe, while in the more distant one FIR emission is extremely common amongst radio-active AGN.

Furthermore, as shown by [11], also the distribution of radio spectral indices of radio-selected AGN looks identical, irrespective of the fact that the AGN also show emission at FIR wavelengths. This strongly suggests that, at least at radio wavelengths and in the more distant universe, the central engine responsible for the AGN phenomenon is oblivious to the FIR activity which takes place within the same host galaxy or, in other words, that FIR processes do not influence the activity of the central engine, at least that detected at radio wavelengths.

\section{Results: 2) FIR properties}

If FIR emission is so common amongst high-redshift radio-selected AGN, the question one may want to pose is what is this FIR emission due to. In order to answer this question, we report in 
Figure 3 the distribution of FIR, $\mathrm{F}_{100 \mu \mathrm{m}} / \mathrm{F}_{160 \mu \mathrm{m}}$, colours as a function of redshift for AGN of different radio luminosity. The Figure refers to all the four considered fields, whereby in the right-hand plot small symbols are for GOODS-S, intermediate-size ones for GOODS-N and large symbols are for the Lockman Hole, while the left-hand plot refers to COSMOS. The dashed and dotted lines represent the Spectral Energy Distributions (SED) of two well studied templates for star-forming galaxies such as M82 (dashed line) and Arp220 (dotted line). It is clear that, irrespective of cosmic age and radio luminosity, and except for very few outliers, the FIR colour distribution of radioactive AGN perfectly follows those of typical moderate-to-intense star-forming galaxies, proving that FIR emission from the hosts of radio AGN is entirely to be attributed to star-forming processes within the galaxy itself.
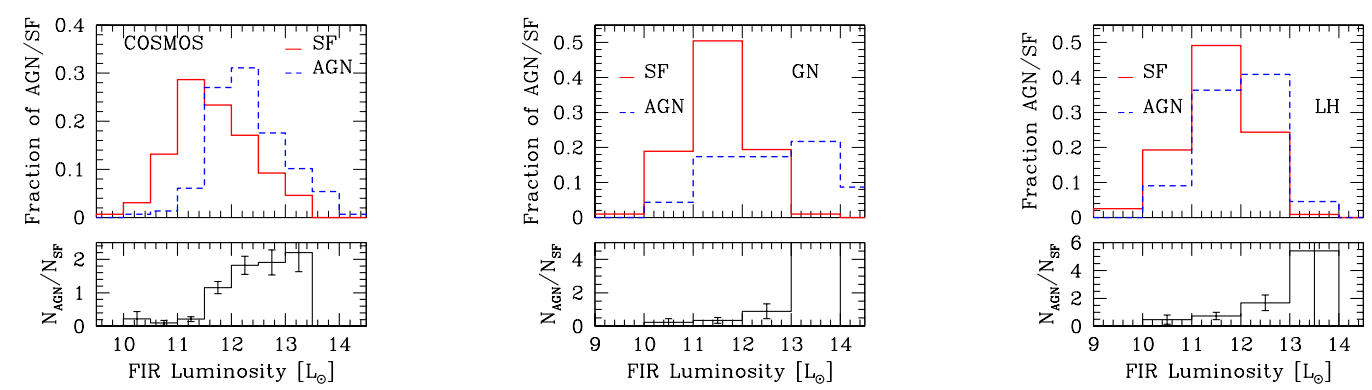

Figure 4: FIR luminosity distribution for sources in the COSMOS field (left-hand panel), GOODS-N (middle panel) and Lockman Hole (right-hand panel).. The solid histograms represent the class of radio-selected star-forming galaxies, while the dashed ones that of radio-selected AGN. The bottom panels show the ratio between the two distributions, normalized by the total number of AGN and SF in each field.

So, we have shown that FIR emission from radio-selected AGN originates from star-forming processes within the host galaxy. But how bright are these sources at FIR wavelengths? And what are their star-formation rates? In order to provide such answers, we have then estimated the total IR luminosities, $\mathrm{L}_{\mathrm{IR}}$, of those radio-active AGN which showed a counterpart in the Herschel maps. These are shown in Figure 4. Our data clearly indicate that radio-active AGN are extremely luminous sources: their bolometric luminosities extend up to $\sim 10^{13}-10^{14} \mathrm{~L}_{\odot}$, which in turn correspond to extremely high star-formation rates, SFR $\gtrsim 10^{3} \mathrm{M}_{\odot} \mathrm{yr}^{-1}$. These results imply that galaxies hosts of radio AGN activity are indeed also the sites of extremely intense emission of star-forming origin. AGN activity does not seem to inhibit star formation within the host galaxy, just as FIR activity does not seem to affect radio AGN luminosity, at least in the relatively distant $(z \gtrsim 1, \mathrm{cfr} \S 3)$ universe. Given the extremely high rate of associations which, we repeat, approaches $\gtrsim 70 \%$ in deep fields such as GOODS-N, we stress that the above conclusions do not simply refer to a limited sub-sample of radio-active AGN but rather include the overwhelming majority of the considered sources: most radio-active AGN are associated with intense episodes of star-formation. However, the two processes proceed independently within the same galaxy, at all redshifts except 

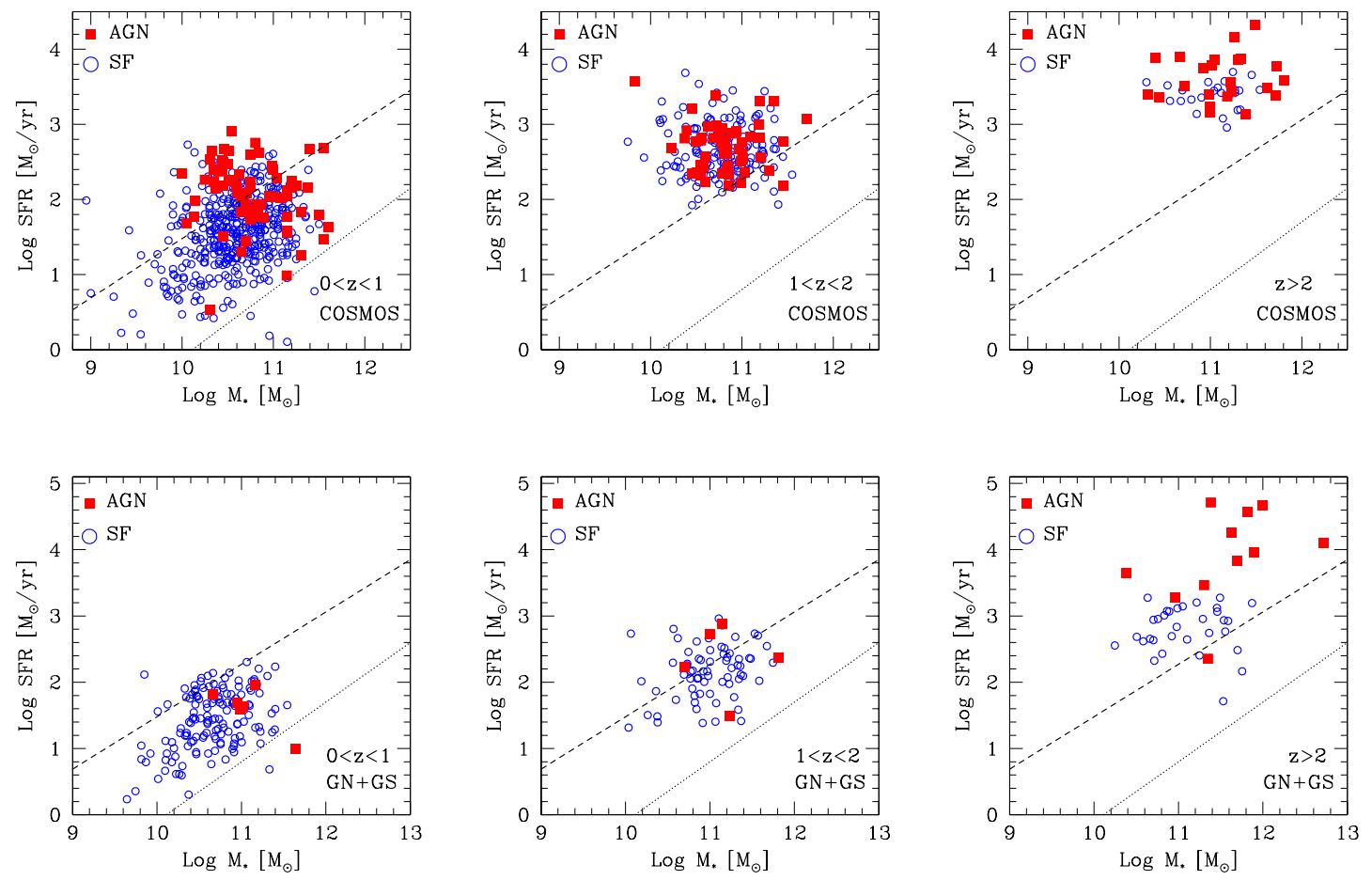

Figure 5: Distributions of stellar masses vs star-formation rates for radio-selected sources which are also FIR-detected in the COSMOS (upper panels) and in the combined GOODS-N+GOODS-S fields (lower panels). Filled squares represent AGN, while open circles show star-forming galaxies. From left to right, different panels refer to different redshift intervals. The dashed lines indicate the relation obtained for main sequence galaxies at $\mathrm{z} \sim 2$ by [14], while the dotted lines that derived for local galaxies by [15].

in the most local universe, where possibly intense radio activity acquires enough power to switch off the on-site star formation.

Not only that. Figure 4 also clearly shows that the distribution of FIR luminosities for radioselected sources which contain a central radio-active AGN is markedly different from that of those galaxies which do not. First of all, AGN are much more FIR-bright objects. The overwhelming majority of these sources indeed exhibits star-formation rates above $\sim 100 \mathrm{M}_{\odot} \mathrm{yr}^{-1}\left(L_{\mathrm{IR}} \gtrsim 10^{11}\right.$ $\mathrm{L}_{\odot}$ ), while star-forming galaxies are also found down to very low IR luminosities (of the order of a few $10^{10} \mathrm{~L}_{\odot}$ ). At the same time, the luminosity distribution of radio-selected AGN extends up to extremely bright luminosities, while that of star-forming galaxies stops at around $10^{3} \mathrm{M}_{\odot} \mathrm{yr}^{-1}$ (with slightly higher values observed in COSMOS which, we remind, includes on average brighter FIR sources due to the lower sensitivity of Herschel observations both at $100 \mu \mathrm{m}$ and $160 \mu \mathrm{m}$ ).

So, are these differences between radio-selected AGN and star-forming galaxies inbuilt in the process of their formation or do these two populations differentiate at a certain point of their evolution? The answer can be found in the plots presented in Figure 5. These show that, a part from a number of outliers which mostly represent objects set at very high $(\mathrm{z} \gtrsim 4)$ redshifts and mainly lie in the combined GOODS-S+GOODS-N fields, the distributions of radio-selected AGN and star-forming galaxies are almost indistinguishable one from the other down to $\mathrm{z} \sim 1$. The two 
populations only differentiate in the most local universe, whereby AGN are on average endowed with much larger masses and luminosities than star-forming galaxies.

These results are in agreement and extend the findings of the work presented in $\S 3$ which indicate that for redshifts above $\sim 1$ there is no difference between radio-selected AGN and the sub-population of those which are also active at FIR wavelengths. This latest analysis further shows that, except for a few cases which mostly reside in the very early universe, at the same high redshifts there is also no difference between FIR-active, radio-selected AGN and FIR-active,radio-selected, star-forming galaxies. In all cases, differences appear only in the local universe: radio-active AGN start progressively becoming FIR-quiet, the more as the higher is their radio luminosity. At the same time, AGN are found associated to much higher galaxy masses than star-forming galaxies. Interestingly enough, the few AGN which still emit at FIR wavelengths in the local universe exhibit higher FIR luminosities than star-forming galaxies belonging to the same mass range (cfr left-hand plots of Figure 5).

\section{References}

[1] Bondi M. et al. 2003, A\&A, 403, 857

[2] Ilbert O., 2013, A\&A, 556, 55

[3] Ibar E., Ivison R.J., Biggs A.D., Lal D.V., Best P.N., Green D.A., 2009, MNRAS, 397, 281

[4] Fotopoulou S. et al., 2012, ApJS, 198, 1

[5] Morrison G.E., Owen F.N., Dickinson M., Ivison R.J., Ibar E., 2010, ApJS, 188, 178

[6] Berta S. et al., 2011, A\&A, 532, 49

[7] Miller N.A. et al., 2013, ApJS, 205, 13

[8] Santini P. et al., 2009, A\&A, 504, 751

[9] Lutz D. et al., 2011, A\&A, 532, 90

[10] Magliocchetti M. et al., 2014, MNRAS, 442, 682

[11] Magliocchetti M. et al., 2016, MNRAS, 456, 431

[12] McAlpine K., Jarvis M.J., Bonfield D.G., 2013, MNRAS, 436, 1084

[13] Magliocchetti M. et al. (the 2dFGRS Team) 2002, MNRAS, 333, 100

[14] Rodighiero G. et al. 2011, ApJ, 739, L40

[15] Brinhcmann J., Charlot S., White S.D.M. Tremonti C., Kauffmann G., Heckman T., Brinkman J., 2004, MNRAS, 351, 1151 